\title{
Does the use of locking plates or mesh and wires influence the risk of symptomatic non-union of the sternal osteotomy after modified Ravitch?
}

\author{
Erik R. de Loos ${ }^{\wedge}$, Karel W. E. Hulsewé ${ }^{1}$, Enzo R. J. van Loo ${ }^{1}$, Johannes A. Kragten ${ }^{2}$, Paul F. Höppener ${ }^{2}$, \\ Jamiu O. Busari ${ }^{3}$, Yvonne L. J. Vissers ${ }^{1}$ \\ ${ }^{1}$ Department of General and Thoracic Surgery, ${ }^{2}$ Department of Cardiology, ${ }^{3}$ Department of Pediatrics, Zuyderland Medical Center, Heerlen, The \\ Netherlands \\ Contributions: (I) Conception and design: ER de Loos, PF Höppener, KWE Hulsewé, YLJ Vissers; (II) Administrative support: ERJ van Loo, PF \\ Höppener; (III) Provision of study materials or patients: ER de Loos, KWE Hulsewé, PF Höppener, JO Busari, KWE Hulsewé, YLJ Vissers; (IV) \\ Collection and assembly of data: ER de Loos, ERJ van Loo, YLJ Vissers; (V) Data analysis and interpretation: ER de Loos, KWE Hulsewé, YLJ \\ Vissers; (VI) Manuscript writing: All authors; (VII) Final approval of manuscript: All authors. \\ Correspondence to: Enzo R. de Loos, MD. Department of General Surgery, Division of Thoracic Surgery, Zuyderland Medical Center, PO BOX 5500, \\ NL-6130 MB Sittard-Geleen, The Netherlands. Email: e.deloos@zuyderland.nl.
}

Background: Patients with pectus excavatum which is unsuitable for minimally invasive repair are usually treated by modified Ravitch procedure. For fixation of the sternal osteotomy, mesh and wires are mostly used. To decrease non-union risk, we introduced a technique with double locking plate fixation of the osteotomy and compared this to fixation using mesh and wires.

Methods: Patients undergoing a modified Ravitch procedure for pectus excavatum between 2006 and 2016 were included. From 2006 to 2012, the sternum was fixated with mesh and wires. From 2012 to 2016, locking compression plates (LCP) were used. Baseline parameters, symptomatic non-union and total number of complications were retrospectively compared. Statistical analysis was performed using Mann-Whitney or Fisher's exact test. Data are presented as means +/- SD.

Results: Forty-four patients were included. In 18 patients, the sternum was fixed with mesh and wires, in 26 patients with LCP. Mean follow-up was 35 months in the mesh and 30 months in the LCP group, $\mathrm{P}=0.71$. Haller index was similar in both groups (mesh $3.8 \pm 1.3$ vs. LCP $3.9 \pm 1.1, \mathrm{P}=0.81$ ). Symptomatic nonunion occurred in $17 \%(n=3)$ in the mesh group and did not occur after LCP, $P=0.062$. Total number of complications was $33 \%(n=6)$ in the mesh group and $15 \%(n=4)$ after LCP, $P=0.27$.

Conclusions: After modified Ravitch procedure, union of the sternal osteotomy is challenging. In this retrospective cohort study, a lower incidence of symptomatic non-union was observed after fixation of the sternum with LCPs, with a trend towards statistical significance.

Keywords: Pectus excavatum; modified Ravitch; locking compression plate osteosynthesis (LCP osteosynthesis); non-union; sternal osteotomy.

Submitted Jan 14, 2020. Accepted for publication May 28, 2020.

doi: $10.21037 /$ jtd-20-527

View this article at: http://dx.doi.org/10.21037/jtd-20-527

\footnotetext{
$\wedge$ ORCID: 0000-0001-6313-2658.
} 


\section{Introduction}

Pectus excavatum is a common deformity of the anterior thoracic wall. Pectus excavatum represents $90 \%$ of the congenital chest wall deformities and is associated with connective tissue diseases as Marfan syndrome or EhlersDanlos syndrome (1). The severity of the pectus is classically calculated by the Haller index, which divides the distance at the widest point of the inner chest by that between the sternum and the vertebral column (2).

Anterior chest wall deformities can lead to a variety of symptoms, including adverse cosmetic and psychological effects and physiological impairment. In pectus excavatum, shortness of breath, palpitations and reduced exercise intolerance are frequently reported complaints, caused by cardiac impression due to the depressed sternum. Surgical correction may improve cardiovascular function and quality of life (3).

Elderly patients with an untreated pectus excavatum form a special category. Until recently, symptomatic pectus excavatum in seniors was considered non-existent. Nowadays, symptomatic pectus excavatum in seniors is more and more seen as a clinically relevant cardiovascular disease $(4,5)$. In selected cases, surgical correction results in long term increase of cardiovascular function and quality of life (6).

In the past decades, many techniques have been developed for operative correction of pectus excavatum. The most well-known is the Ravitch procedure, which was introduced in 1949. In the original Ravitch procedure, perichondrium and all deformed costal cartilage are removed and the xiphoid process is detached. A transverse cuneiform osteotomy is performed at the sterno-manubrial junction and the corrected position is maintained by mattress sutures of braided silk. The periosteum is then sutured with black silk (7). Over the years, small modifications to the original operation have been reported, such as preservation of the perichondrium $(8,9)$ and preserving of the attachment of the xiphoid. The technique currently used, is unchanged in essence and includes a transverse correction osteotomy of the sternum after resection of all affected costal cartilage. For reinforcement, Kirschner wires, cerclage wires, struts or non-absorbable meshes are used. However, all these techniques provide only relative stability. This can lead to complications such as pain and non- or mal-union of the osteotomy due to instability.

Discomfort and disability due to sternal healing problems are common findings after sternotomy. Risks of post-sternotomy instability after cardiac surgery have been reported in up to $8 \%$ of patients (10). It is likely that the incidence of symptomatic pseudarthrosis is substantially higher after Ravitch than medial sternotomy for cardiac surgery due to extensive tissue dissection and added instability after cartilage resection. Furthermore, forces on the corrected sternum after pectus repair are expected to be greater than those after cardiac surgery due to the aberrant form of the chest wall. However, to the best of our knowledge, no data has been published on the incidence of non-union after Ravitch.

To achieve rigid fixation of the sternal osteotomy in Ravitch, plate fixation could be an alternative to conventional techniques. Locking compression plates (LCP) are nowadays considered to be the gold standard for plate osteosynthesis in fracture surgery. This stable plate-screw connection leads to excellent stability of the implant and reduces the risk of primary or secondary loss of reduction and screw loosening. Theoretically, this leads to less post-operative pain and a decrease in the risk of non- or malunion.

The aim of this retrospective cohort study is to evaluate the use and outcome of a new fixation method of the sternal osteotomy in the modified Ravitch procedure using LCPs. We compare this technique to the standard fixation method, during which cerclage wires with non-absorbable retrosternal mesh support are used. We present the following article in accordance with the STROBE reporting checklist (11) (available at http://dx.doi.org/10.21037/jtd20-527).

\section{Methods}

\section{Patient work-up}

Zuyderland Medical Center is a large non-university teaching hospital in the south of the Netherlands and serves as a tertiary referral center for pectus and thoracic wall surgery. All pectus patients are seen in a multidisciplinary setting, including participation of specialized general thoracic surgeons, paediatricians and cardiologists. Standard work-up includes chest $\mathrm{x}$-rays and imaging by a medical photographer (Figure 1). Besides normal photographs, the depth of the excavation is measured with a ruler (Figure 2) and a 3-D grid-projection (grid stereogram) is made (Figure 1B) (12). On indication, CT- and MRI-scans are performed (Figure 3). Cardiac workup includes electrocardiography, echocardiography and dynamic cardiac 

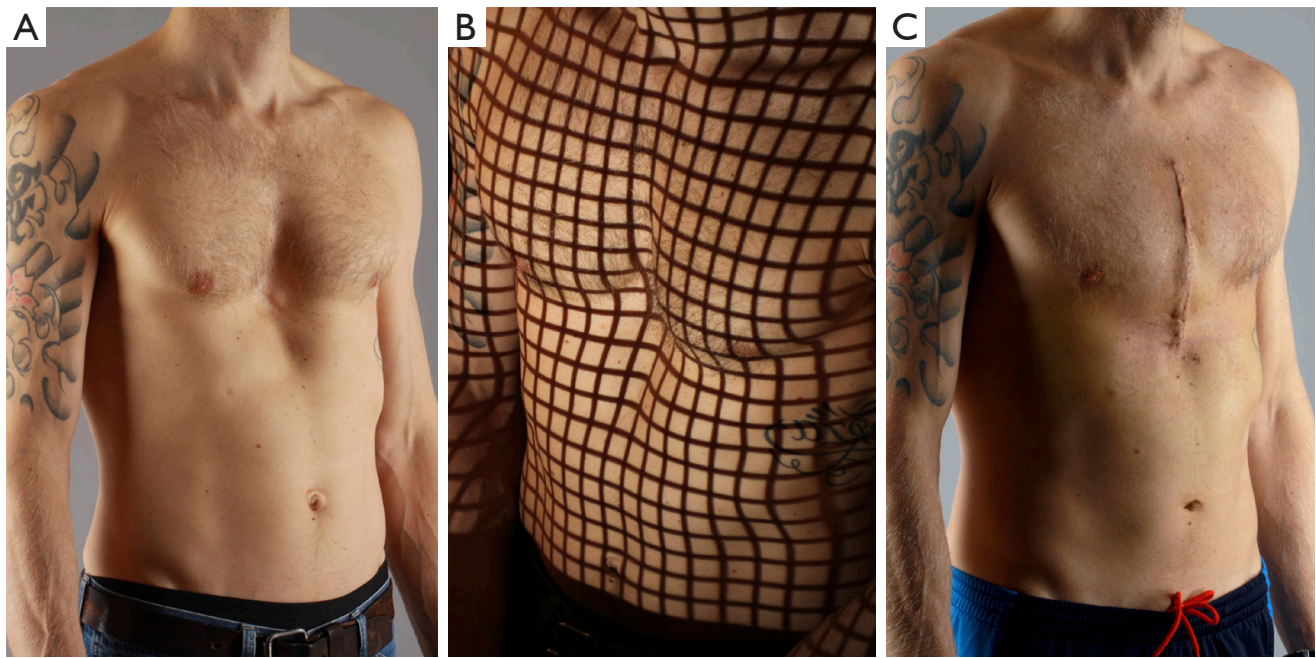

Figure 1 Preoperative imaging (A), 3D grid stereogram (B) and postoperative imaging (C) by medical photographer.

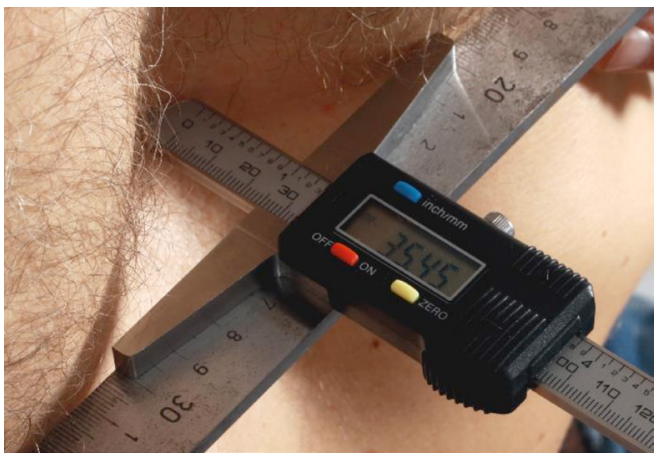

Figure 2 Preoperative depth measurement of excavation.

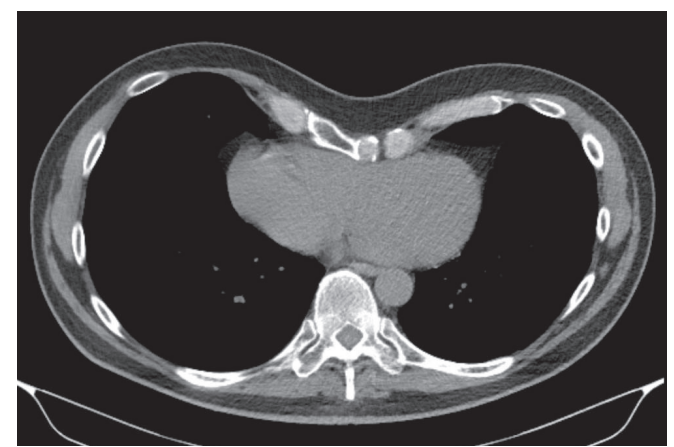

Figure 3 Preoperative CT-scan with cardiac impression due to depressed sternum.

MRI. When palpitations are part of the symptomatology, holter recordings are done. If necessary, pulmonary function tests including triflow forced inspiration tests are conducted (13). Indications for surgical intervention are: (I) impression of the pectus on the right ventricle of the heart established by echocardiography or MRI; (II) exercise intolerance, c. arrhythmias, d. psychosocial problems due to aesthetic complaints.

The vast majority of our patients is young, suffers from a symptomatic pectus excavatum with exercise intolerance and is treated with a minimally invasive thoracoscopic Nuss bar procedure (MIRPE). Some younger patients are unsuitable for MIRPE and therefore are treated with a modified Ravitch procedure. This could be due to prior pleurodesis or thoracic surgery, or morphological deformities as extreme bending or torsion of the sternum (e.g., banana shape deformity) warranting open correction, or on specific patient request. In seniors with a symptomatic pectus excavatum, decision between MIRPE and open repair is based on expected chest wall rigidity and patient preference.

\section{Study methods}

All patients undergoing a modified Ravitch procedure for pectus excavatum at our institution between March 2006 and September 2016 were included in this retrospective cohort study. Selection of the two surgical techniques was based on historical grounds. From March 2006 to March 2012, the sternum was fixated with cerclage wires and nonabsorbable retrosternal mesh support as standard of care. From March 2012 to September 2016, LCPs were used for fixation. In the latter group, no additional retrosternal mesh was used. Data were extracted from the electronic patient files. The study was approved by the local ethics and clinical 


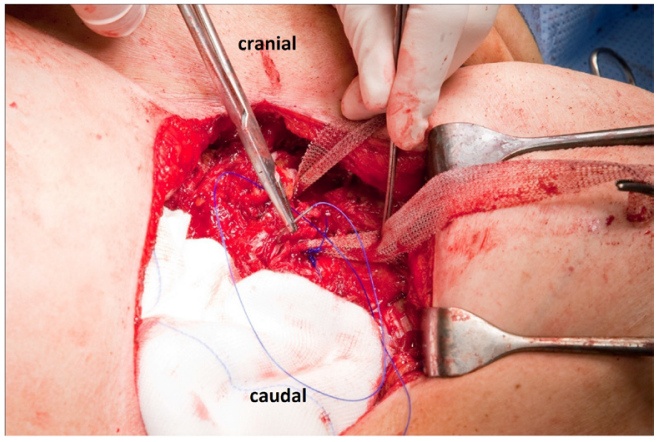

Figure 4 Intra-operative result: retrosternal mesh support.

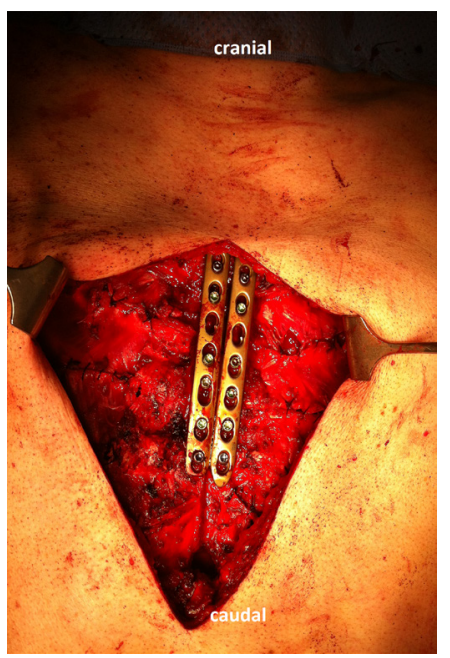

Figure 5 Intra-operative result: locking compression plate osteosynthesis.

research committee, waiving the need for individual patient consent (METC Zuyderland, ID: METCZ20200049). The study was conducted in accordance with the Declaration of Helsinki and the Harmonized Tripartite Guideline for Good Clinical Practice from the International Conference on Harmonization. The following baseline and perioperative parameters were recorded: age, gender, cardiac impression, Haller index, operation time, blood loss, hospital stay. The standard follow-up consisted of taking history and physical examination. There was no mandatory follow-up regime with regards to frequency and duration. Visits to the outpatient clinic were scheduled according to physicians' judgement and patients' preferences. When clinical signs of non-union were present, for example excessive pain of subjective instability, a CT-scan was performed for definitive diagnosis of non-union.
Thus, symptomatic non-union was defined as clinical symptoms of excessive pain or instability after at least 6 months follow-up, combined with confirmation of the non-union by CT scan. All post-operative complications were analysed. Primary outcome measure was symptomatic non-union of the sternal osteotomy. Secondary outcome was the total number of complications.

\section{Surgical technique}

The patient was placed in supine position. The operation was performed under epidural and general anaesthesia with double lung ventilation. Antibiotic prophylaxis was used. Longitudinal midline or inframammary incisions were used for the approach. Full thickness subcutaneous flaps were created. The pectoralis muscles were dissected and lifted off the sternum and anterior thoracic wall. In all deformed ribs, the perichondrium was elevated and deformed rib cartilage was removed with preservation of the perichondrium. The sternum was lifted and a transverse wedge osteotomy was made on the upper margin of the sternal deformity. The sternum was then anatomically reduced. In the mesh support group, the sternal osteotomy was fixated with cerclage wires and non-absorbable retrosternal mesh support (Marlex ${ }^{\circledR}$, Bard medical, IJsselstein, the Netherlands) (Figure 4). In the LCP group, the osteotomy was rigidly fixated by two 3.5 millimeter 6 to 8 -hole metaphyseal or diaphyseal titanium LCPs (DePuy Synthes, Johnson\&Johnson, Amersfoort, the Netherlands) (Figure 5). Finally, the wound was closed in layers after low-vacuum drain placement.

\section{Statistics}

For statistical analysis SPSS version 23 was used (IBM Corp. Released 2015. IBM SPSS Statistics for Windows, Version 23.0. Armonk, NY: IBM Corp). Differences between groups were tested for significance using MannWhitney test or Fisher's exact test where appropriate. A P value of $<0.05$ was considered statistically significant. Data are presented as means $+/-$ SD. Missing data and data lost to follow-up were omitted from the analyses.

\section{Results}

Baseline and perioperative characteristics are listed in Table 1. A total of 44 patients (28 men, 16 women) were included. One patient was lost to follow-up after two months. Median age was 56 years in the mesh group (range, 19-72) and 
Table 1 Baseline characteristics

\begin{tabular}{|c|c|c|c|c|c|c|c|}
\hline Characteristics & \multicolumn{3}{|c|}{ Mesh, $n=18$} & \multicolumn{4}{|c|}{ Locking compression plate, $n=26$} \\
\hline Age (years) & 56 (median 56) & $19-72$ & 11 & 46 (median 48) & $20-72$ & 15 & $0.028^{*}$ \\
\hline Gender & & & & & & & $0.76^{\#}$ \\
\hline Male & $12(67 \%)$ & & & $16(62 \%)$ & & & \\
\hline Cardiac impression & $18(100 \%)$ & & & $22(85 \%)$ & & & 0.13 \\
\hline Haller index & 3.8 & $2.1-6.5$ & 1.3 & 3.9 & $1.9-6.4$ & 1.1 & $0.81^{*}$ \\
\hline Operation time (min) & 141 & $60-210$ & 39.5 & 147 & $56-180$ & 33 & $0.48^{*}$ \\
\hline Blood loss (mL) & 275 & $0-500$ & 263 & 227 & $0-600$ & 152 & $0.78^{*}$ \\
\hline
\end{tabular}

Differences between groups were tested for significance using: *Mann-Whitney test or "Fisher's exact test where appropriate. A P value of $<0.05$ was considered statistically significant.
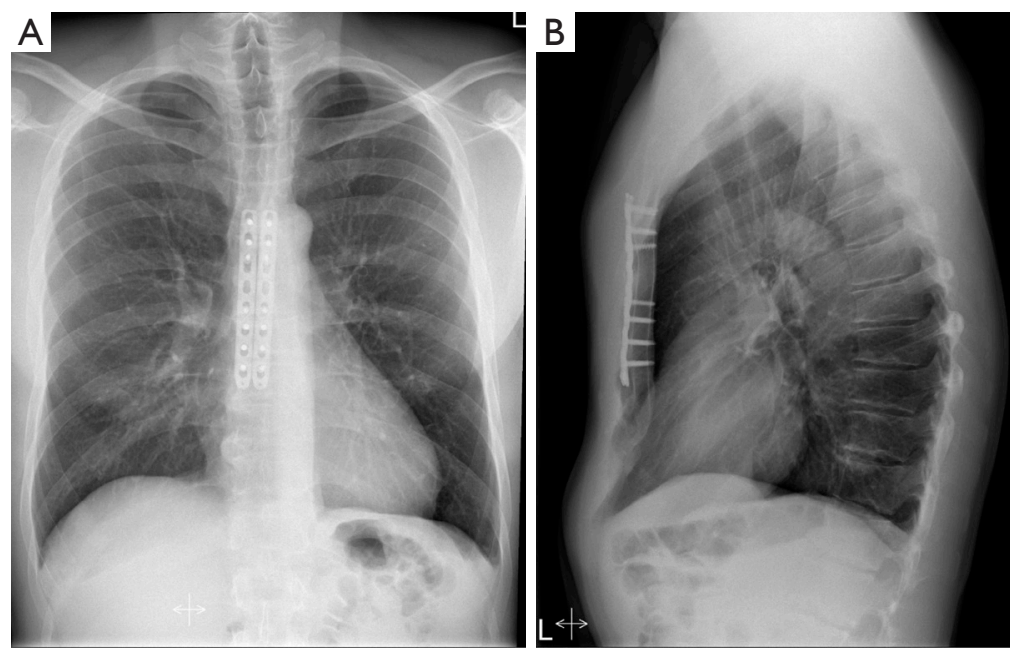

Figure 6 Postoperative chest X-ray after locking compression plate osteosynthesis.

48 years in the LCP group (range, 20-72), $\mathrm{P}=0.028$. The depth of the pectus measured as Haller index was similar in both groups (mesh $3.8 \pm 1.3 v s$. LCPs $3.9 \pm 1.1, \mathrm{P}=0.81$ ).

In 18 patients $(41 \%)$ the sternum was fixed with mesh support and in 26 patients (59\%) with LCPs. The mean follow-up was 35 months in the mesh group (range, 8-83) and 30 months in the LCP group (range, 2-73), $\mathrm{P}=0.71$. There was no significant difference between groups with regard to the presence of cardiac impression, operation time, blood loss or hospital stay (Table 1).
Symptomatic non-union occurred in three patients in the mesh group (17\%) compared to zero in the locking compression group, $\mathrm{P}=0.062$. Patients presented with pain or tenderness of the sternum, and all had radiological signs of non-union on CT. During follow-up, no failures of the osteosynthesis were recorded in the LCP group (Figures 6,7). On patient request, plates were uneventfully removed in $6 / 26$ cases $(23 \%)$. All these patients were bothered by implant prominence and therefore asked for hardware removal. No implants had to be removed due to infection 

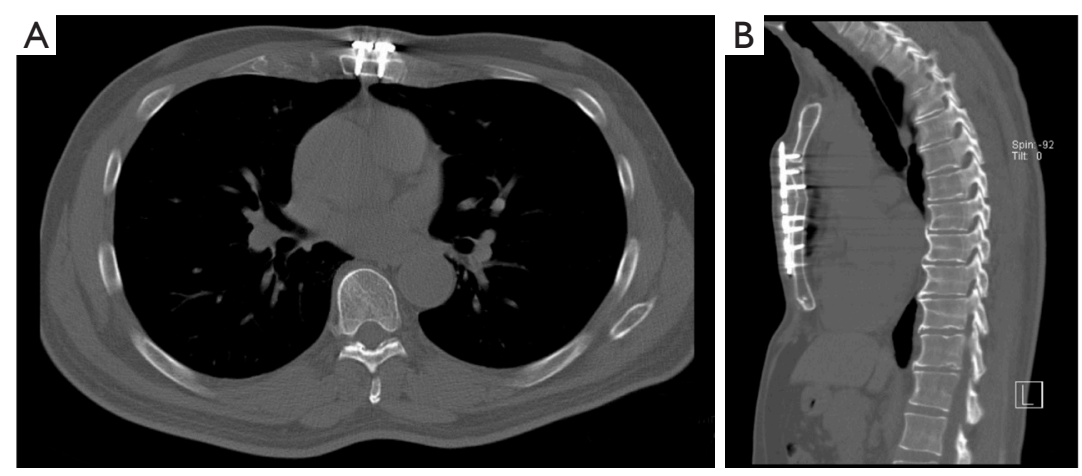

Figure 7 Postoperative CT-scan after locking compression plate osteosynthesis.

Table 2 Postoperative complications

\begin{tabular}{lccc}
\hline Variable & Mesh, $\mathrm{n}=18$ & Locking compression plate, $\mathrm{n}=26$ & $\mathrm{P}$ \\
\hline Symptomatic non-union & $3(17 \%)$ & $0(0 \%)$ & 0.062 \\
Hardware removal & 0 & $6(23 \%)$ & 0.067 \\
Complications other than above & $6(33 \%)$ & $4(15 \%)$ & 0.27 \\
\hline
\end{tabular}

Differences between groups were tested for significance using Fisher's exact test. A P value of $<0.05$ was considered statistically significant.

or pain. The total number of complications other than hardware removal or non-union was $33 \%$ in the mesh group ( $\mathrm{n}=6)$ and $15 \%$ in the LCP group $(\mathrm{n}=4), \mathrm{P}=0.27$ (see Table 2). More specifically, in the mesh group: one patient presented with an incisional hernia, one patient with chronic pain, two patients with pneumonia, one patient with pericardial effusion, and one patient with atrial fibrillation. In the locking plate group: one patient presented with pectoral muscle dehiscence, one patient with seroma and two patients with wound infections.

\section{Discussion}

In this retrospective cohort study in patients with pectus excavatum undergoing repair by a modified Ravitch procedure, a lower incidence of symptomatic non-union was observed after fixation of the sternum with LCPs, with a trend towards statistical significance.

Challenges in the Ravitch technique are preservation of the corrected position of the sternum and achieving union of the osteotomy. Many modifications have been developed to create more stability. Temporary metal bars such as Rehbein pins or Nuss bars are frequently used for extra retrosternal support. Fonkalsrud describes good results with a less invasive technique including minimal cartilage resection and retrosternal support with an Adkins strut, which was routinely removed within 6 months (14). Other surgeons use a non-absorbable Marlex mesh. Fixation of the osteotomy can be done with Kirschner wires, cerclage wires or trans-osseous sutures. Limitations of these techniques are the provided relative stability, increasing the risk of mal-union or non-union, as well as the need for implant removal in case of temporary fixation which leads to a high reoperation rate. A recent article describes a $100 \%$ failure rate of using a combination of steel wires and substernal STRATOS bars in 12 patients, all requiring reoperation (15).

To enable physiological bone healing, stability at the site of the osteotomy is important, whereas the Ravitch procedure by itself creates a situation of instability of the sternum. Therefore, an alternative to conventional techniques could be the use of LCPs, as these are known for their excellent stability in the field of fracture surgery. These plates are manufactured in a wide variety of shapes and diameters for use in different body areas. The key concept is the plate design which provides a low-profile footprint and combination holes for both standard and locking screw placement. Angular stability is created by the rigid fixation of the tread on the head of a locking screw in the treaded part of the combination hole in the plate. Until now, literature on the use of plate fixation in sternal 
osteotomies for pectus repair is limited to a few case reports. Raff $e t a l$. reported the use of a sternal plate in a revision operation of a patient with sternal erosion of a Nuss bar (16). Pasrija et al. used the combination of a sternal H-plate and 4 rib plates in correction of a recurrent pectus excavatum in a 39-year-old patient (17). Puma et al. describe a surgical technique with two longitudinal thin titanium struts (18). Ours is the first cohort study retrospectively comparing groups with various fixation regimes for the sternal osteotomy in modified Ravitch procedures.

Non-union can be assessed in various ways. Radiological evidence of non-union consists of widening of the fracture gap and hypertrophic or absent callus formation. Due to the retrospective nature of our series, radiological bone healing was not standardly assessed in all patients. However, in general non-union will only be treated if clinical sequelae occur, with pain and local tenderness being the most reported complaints. Therefore, we think it is more relevant to focus on clinically evident failure of bone healing.

Per definition, non-union occurs when consolidation fails after six months. One patient in our series was lost to follow-up after two months. All other patients were followed at least 8 months.

Although often multifactorial, the most important factors leading to non-union and pseudarthrosis are instability, disturbed vascularity and infection. There were no significant differences in comorbidities related to mal-union, such as cardiovascular disease and diabetes. Moreover, the number of smokers was similar in both groups. Therefore, we assume that there are no relevant confounders for the observed difference in non-union.

The plates we used up to now are rather bulky. This thick material provides optimal strength to bear the high forces on chest wall and sternum. A disadvantage however, is that the plates lie superficially underneath the pectoralis muscles and thin overlying subcutaneous tissues and are sometimes palpable, especially in lean patients. Six patients experienced burden from implant prominence and have therefore asked for hardware removal. No implants had to be removed due to infection or pain. In the future it may be possible to use firm but less thick plates to fixate the osteotomy of the sternum. Recently, specific sternal plates have become available, which could be an interesting alternative to conventional LCP.

To the best of our knowledge, this is the first comparative study using LCPs in the modified Ravitch procedure. In our experience, anatomical reduction of the sternal correction osteotomy on the plates is easy to achieve. The double locking plate technique provides excellent stability. Theoretically, this leads to less post-operative pain and a decrease in the risk of non- or mal-union. Our data suggest a lower incidence of symptomatic non-union with a trend towards statistical significance in the group treated with LCPs. However, further experience is necessary to support this new technique.

There are several limitations to this retrospective cohort study. Complications were only identified on clinical presentation. Therefore, potential asymptomatic non-unions could have been missed, resulting in an underestimation of the total number of non-unions. However, re-intervention is only mandatory in presence of symptoms of non-union, like pain, discomfort or hardware failure. The non-union rate reported in our study may thus represent clinically relevant non-unions only. Another disadvantage of the retrospective aspect of our study is the variance in follow-up. According to our protocol, postoperative patients are seen in our outpatient department after 2 and 6 weeks, 3, 6, 12, 18 and 24 months. In practice, actual follow-up ranged from 2 to 83 months, potentially biasing our results. There was only one patient with a follow-up of less than 8 months. Thus, the vast majority of patients had a follow-up of more than 6 months, which is the minimum required for diagnosing non-union.

Furthermore, this is a relatively small single center retrospective experience accumulated over many years, during which time there could have been a number of opportunities for selection bias and other factors which might have modified results. On the other hand, previously published series describing open repair of pectus excavatum are relatively small. Therefore, we think that our study attributes to provide an insight on incidence and preventive strategies for non-union in modified Ravitch procedures.

In our goal to achieve absolute stability, we used relatively thick conventional LCPs. Adding mechanical stability led to a reduction in symptomatic non-union. However, the price paid is the occurrence of implant related symptoms, relative frequently leading to reoperation for removal of the osteosynthesis material. Therefore, we recently started using less thick specific sternal plates for angular stable fixation of the sternal correction osteotomy. Long-term follow-up is necessary to investigate symptomatic nonunion rate and need for implant removal. If results of less thick and smaller plates prove to be favourable, percentage of re-operation for hardware removal and herewith the total complication rate could further be reduced.

In conclusion, double locking plate fixation of the 
sternal osteotomy is a feasible alternative in the modified Ravitch procedure. In this retrospective cohort study, we noted promising initial clinical results with the use of LCPs instead of mesh and wires for sternal fixation. Our data suggest a lower incidence of symptomatic non-union with a trend towards statistical significance in the group treated with LCPs.

\section{Acknowledgments}

Funding: None.

\section{Footnote}

Reporting Checklist: The authors have completed the STROBE reporting checklist. Available at http://dx.doi. org/10.21037/jtd-20-527

Data Sharing Statement: Available at http://dx.doi. org/10.21037/jtd-20-527

Peer Review File: Available at http://dx.doi.org/10.21037/jtd20-527

Conflicts of Interest: All authors have completed the ICMJE uniform disclosure form (available at http://dx.doi. org/10.21037/jtd-20-527). The authors have no conflicts of interest to declare.

Ethical Statement: The authors are accountable for all aspects of the work in ensuring that questions related to the accuracy or integrity of any part of the work are appropriately investigated and resolved. The study was conducted in accordance with the Declaration of Helsinki (as revised in 2013) and the Harmonized Tripartite Guideline for Good Clinical Practice from the International Conference on Harmonization. The study was approved by the local ethics and clinical research committee, waiving the need for individual patient consent (METC Zuyderland, ID: METCZ20200049).

Open Access Statement: This is an Open Access article distributed in accordance with the Creative Commons Attribution-NonCommercial-NoDerivs 4.0 International License (CC BY-NC-ND 4.0), which permits the noncommercial replication and distribution of the article with the strict proviso that no changes or edits are made and the original work is properly cited (including links to both the formal publication through the relevant DOI and the license). See: https://creativecommons.org/licenses/by-nc-nd/4.0/.

\section{References}

1. Koumbourlis AC. Pectus excavatum: pathophysiology and clinical characteristics. Paediatr Respir Rev 2009;10:3-6.

2. Haller JA Jr, Kramer SS, Lietman SA. Use of CT scans in selection of patients for pectus excavatum surgery: a preliminary report. J Pediatr Surg 1987;22:904-6.

3. Handels N, Kragten JA, de Loos E, et al. Long-term results of surgical correction of symptomatic pectus excavatum in seniors: improvement in cardiovasculair function and quality of life. Ital J Vasc Endovasc Surg 2017;24:131-6.

4. Kragten HA, Siebenga J, Höppener PF, et al. Symptomatic pectus excavatum in seniors (SPES): a cardiovascular problem?: A Prospective cardiological study of 42 senior patients with a symptomatic pectus excavatum. Neth Heart J 2011;19:73-8.

5. Winkens RA, Guldemond FI, Höppener PF, et al. Symptomatic pectus excavatum in seniors: an exploratory study on clinical presentation and incidence in daily practice. ISRN Family Med 2013;2013:373059.

6. Handels NA, Kragten HA, de Loos ER, et al. Longterm results of surgical correction of symptomatic pectus excavatum in seniors: Improvement in cardiovascular function and quality of life. Ital J Vasc Endovasc Surg 2017;24:131-6.

7. Ravitch MM. The Operative Treatment of Pectus Excavatum. Ann Surg 1949;129:429-44.

8. Baronofsky ID. Technique for the correction of pectus excavatum. Surgery 1957;42:884-90.

9. Welch KJ. Satisfactory surgical correction of pectus excavatum deformity in childhood; a limited opportunity. J Thorac Surg 1958;36:697-713.

10. Robicsek F, Fokin A, Cook J, et al. Sternal instability after midline sternotomy. Thorac Cardiovasc Surg 2000;48:1-8.

11. von Elm E, Altman DG, Egger M, et al. The Strengthening in Reporting of Observational Studies in Epidemiology (STROBE) Statement: Guidelines for Reporting Observational Studies. PLoS Med 2007;4:e296.

12. van Dijk H, Hoppener PF, Siebenga J, et al. Medical photography: a reliable and objective method for documenting the results of reconstructive surgery of pectus excavatum. J Vis Commun Med 2011;34:14-21.

13. Höppener PF, Kragten HA, Winkens R. Cardiological aspects of Symptomatic Pectus Excavatum in Adults. In: 
Saxena AK. editor. Chest Wall Deformities. Springer, 2017:261-78.

14. Fonkalsrud EW, Mendoza J. Open repair of pectus excavatum and carinatum deformities with minimal cartilage resection. Am J Surg 2006;191:779-84.

15. Sharma PK, Willems TP, Touw DJ, et al. Implant Failure: STRATOS System for Pectus Repair. Ann Thorac Surg 2017;103:1536-43.

16. Raff GW, Wong MS. Sternal plating to correct an unusual

Cite this article as: de Loos ER, Hulsewé KWE, van Loo ERJ, Kragten JA, Höppener PF, Busari JO, Vissers YLJ. Does the use of locking plates or mesh and wires influence the risk of symptomatic non-union of the sternal osteotomy after modified Ravitch? J Thorac Dis 2020;12(7):3631-3639. doi: 10.21037/jtd-20527 complication of the Nuss procedure: erosion of a pectus bar through the sternum. Ann Thorac Surg 2008;85:1100-1.

17. Pasrija C, Wehman B, Singh DP, et al. Recurrent Pectus Excavatum Repair via Ravitch Technique With Rib Locking Plates. Eplasty 2014;14:ic46.

18. Puma F, Vannucci J, Santoprete S. External longitudinal titanium support for the repair of complex pectus excavatum in adults. Eur J Cardiothorac Surg 2012;42:e166-8. 